\title{
Air nonlinear dynamics initiated by ultra-intense lambda-cubic terahertz pulses
}

\author{
Mostafa Shalaby ${ }^{1, a)}$ and Christoph P. Hauri ${ }^{1,2, a)}$ \\ ${ }^{1}$ SwissFEL, Paul Scherrer Institut, 5232 Villigen PSI, Switzerland \\ ${ }^{2}$ École Polytechnique Fédérale de Lausanne, 1015 Lausanne, Switzerland
}

(Received 19 February 2015; accepted 26 April 2015; published online 7 May 2015)

\begin{abstract}
We report on the measurement of the instantaneous Kerr nonlinearity and the retarded alignment of air molecules $\mathrm{CO}_{2}, \mathrm{~N}_{2}$, and $\mathrm{O}_{2}$ triggered by an intense, lambda-cubic terahertz pulse, a diffractionand transform-limited single-cycle pulse. The strong-field, impulsive low-frequency excitation (3.9 THz) leads to field-free alignment dynamics of these molecules thanks to the terahertz-induced transient dipole moments in the otherwise non-polar molecules. The strong coupling to the terahertz electric transient results in the excitation of coherent large amplitude long-living rotational states at room temperature and ambient pressure. Beyond fundamental investigations of nonlinear properties in gases, our results suggest a route towards field-free molecular alignment at laser intensity well below the ionization threshold. (C) 2015 AIP Publishing LLC. [http://dx.doi.org/10.1063/1.4919876]
\end{abstract}

Air turns into a nonlinear medium for electromagnetic waves under exceptionally strong fields that manifest itself in phenomena like the instantaneous Kerr nonlinearities and retarded molecular alignment. ${ }^{1}$ The minuscule nonlinear response of air has limited the exploration of its nonlinear properties to the optical frequency regime owing to the availability of intense near-infrared (nIR) lasers (e.g., Ti:sapphire with a carrier frequency $\approx 380 \mathrm{THz}$ ). From a fundamental science point of view, nonlinear excitation of air (and other gases) by pulses with a lower carrier frequency, in the socalled terahertz gap $(1-10 \mathrm{THz})$, is of large interest as the dominating quantities, like the Kerr nonlinear coefficient $\left(n_{2}\right)$, are yet unknown. The determination of the air Kerr nonlinear coefficient is essential for prediction and understanding several nonlinear phenomena ${ }^{2}$ such as terahertz (THz) self-guiding (filamentation) and $\mathrm{THz}$ self-phase modulation. Furthermore, such field transients at frequencies in the $\mathrm{THz}$ gap match the natural timescale of rotational molecular dynamics which hold promise for enhanced control of molecular alignment.

Up to now, both the measurement of Kerr nonlinearities and molecular alignment in air at $\mathrm{THz}$ frequencies was hampered by the technological void of intense sources. ${ }^{3-9}$ Fieldfree alignment/orientation of molecules is conventionally done by nIR laser technology which allows for preparing the molecules in a preferred angular distribution prior to their interrogation [Refs. 10 and 11, and references therein]. The elimination of a randomly oriented molecule distribution is beneficial for numerous applications including high harmonic generation, ${ }^{12-14} \mathrm{THz}$ emission, ${ }^{15,16}$ the control of chemical, surface ${ }^{17}$ and photoreactions whose rates depend on the molecular orientation, and others. ${ }^{18-21}$ The most common molecular alignment techniques, based on intense nIR femtosecond laser pulses, may cause unwanted ionization and excitation of other states during the interaction. Molecular orientation/alignment by a $\mathrm{THz}$ pulse offers in

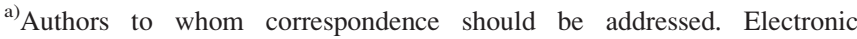
addresses: most.shalaby@gmail.com and christoph.hauri@psi.ch
}

principle superior conditions as the $\mathrm{THz}$ photon quantum energy is 2-3 orders of magnitude smaller than the nIR quantum (meV vs eV), which considerably reduces the probability of multi-photon ionization.

In this work, we report two advances. First, the quantification of the nonlinear Kerr coefficient $\mathrm{n}_{2}$, measured at $\mathrm{THz}$ frequencies and derived from the instantaneous Kerr nonlinearity in air. Second, the measurement of large amplitude induced birefringence due to coherent molecular alignment dynamics of the non-polar nitrogen $\mathrm{N}_{2}$, oxygen $\mathrm{O}_{2}$, and carbon dioxide $\mathrm{CO}_{2}$ in dry air at ambient temperature and pressure by a strong $\mathrm{THz}$ transient. As these molecules do not carry a permanent dipole, the key for the observed alignment dynamics at low $\mathrm{THz}$ frequencies is the recently developed ultra-intense lambda-cubic $\mathrm{THz}$ bullets. ${ }^{22}$ The three orders of magnitude higher $\mathrm{THz}$ field than in previous $\mathrm{THz}$-gas experiments $^{23-25}$ enable the induction of a transient dipole moment leading to a strong transient coupling with the $\mathrm{THz}$ electric field component. Although there is no original research paper on $\mathrm{THz}$-induced molecular alignment, in a review by Hwang et al., ${ }^{26}$ the molecular alignment of $\mathrm{N}_{2}$ and $\mathrm{O}_{2}$ is briefly shown in a figure. However, the authors did not give details on the excitation source, experimental conditions (such as the interaction length), or quantify the measured birefringence. Here, we measure the THz-induced birefringence at similar excitation intensities to those at the optical frequencies. $^{27}$

In order to measure the $\mathrm{THz}$-induced nonlinearity, we performed $\mathrm{THz}$ pump-optical probe spectroscopy (Fig. 1(a)). Our $\mathrm{THz}$ trigger consists of the $\lambda^{3} \mathrm{THz}$ bullet (i.e., a diffraction-limited and transform-limited single-cycle pulse) with maximum electric field strengths of $33 \mathrm{MV} / \mathrm{cm}$ at 3.9 $\mathrm{THz}_{\mathrm{H}}$ center frequency ${ }^{22}$ (Fig. 1(b)). The corresponding peak intensity is $1.44 \mathrm{TW} / \mathrm{cm}^{2}$. The probe pulses are $800 \mathrm{~nm}$ centered pulses polarized at $45^{\circ}$ with respect to the $x$-polarized $\mathrm{THz}$ pump.

The considered air molecules have zero permanent dipole moment and a nearly vanishing average linear susceptibility $\chi^{(1)}$ of $5.4 \times 10^{-4}$. In gases, the third order 
(a)

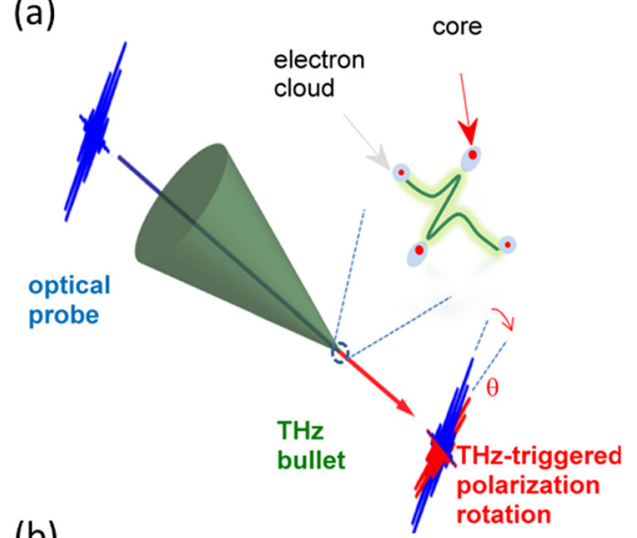

(b)
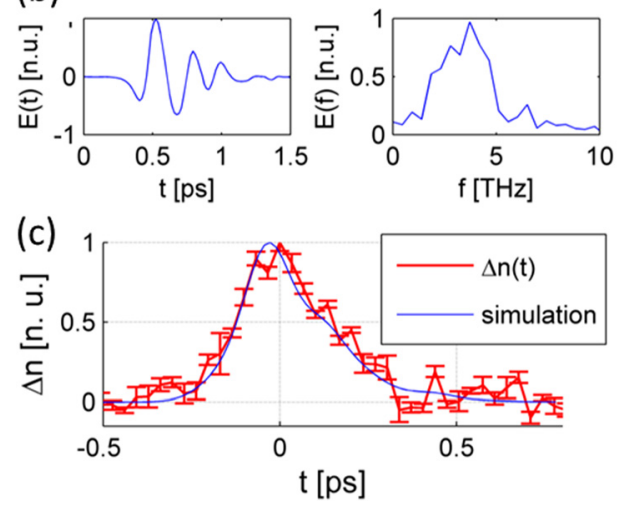

FIG. 1. (a) Schematic diagram of the measurement configuration of the THz-induced nonlinearity. An intense THz bullet nonlinearly induces a transient dipole moment by deforming (displacing) the electron clouds in the air molecules along the $(x-)$ polarization direction of the laser. The near infrared (NIR) probe is initially polarized at $45^{\circ}$ from the $\mathrm{THz}$ polarization. The probe experiences THz-induced birefringence in air. (b) A temporal trace and the corresponding amplitude spectrum of the exciting THz pulse. (c) A comparison between the measured birefringence $\Delta n=n_{x}-n_{y}$ and simulations.

susceptibility $\chi^{(3)}$ is the first nonlinear susceptibility term which is present, due to symmetry constraints. $\chi^{(3)}$ of air is extremely weak, being $5.4 \times 10^{-25} \mathrm{~m}^{2} / \mathrm{V}^{2}$ at the optical frequencies. ${ }^{28}$ Observation of nonlinearities in air requires therefore intensities on the order of $10^{12} \mathrm{~W} / \mathrm{cm}^{2}$. Up to now, such intensities were not available in the THz gap but rather at optical frequencies. At optical frequencies, a multitude of nonlinear phenomena ${ }^{29-31}$ are observed as consequence of coupling the laser electric field to the polarization through $\chi^{(3)}$ and similar phenomena are, in principle, expected at $\mathrm{THz}$ frequencies.

A change in the electronic susceptibility can be experimentally determined by measuring the refractive index $n$. In a centro-symmetric material, they are related ${ }^{28,29}$ by

$$
n=(\chi+1)^{1 / 2}=\left(\chi^{(1)}+1\right)^{1 / 2}+\frac{3 \chi^{(3)}}{4 n_{0}}|E|^{2} / z_{0}=n_{0}+n_{2} I
$$

where $n_{0}$ and $I$ are the linear refractive index and intensity of the exciting field. $n_{2}$ is the nonlinear refractive index, commonly referred to as Kerr coefficient.

The induced birefringence $\Delta n=n-n_{0}=n_{2} I$ is determined from the measurement of the THz induced phase retardation of the probe $\Delta \varphi=\Delta n \omega L / c$, where $\omega$ and $c$ are the optical frequency of the probe and the speed of light in vacuum, respectively. ${ }^{31} I$ is the THz intensity. $L$ is the interaction length. For a tightly focused laser pulse, like our $\mathrm{THz}$ bullet, the $\mathrm{THz}$ intensity changes significantly around the focus. It is thus more accurate to estimate the induced birefringence from $\Delta \varphi=\omega / c \int \Delta n(z) d z$, where $z$ is the propagation direction. As our probe (optical) pulse duration ( $\sim 75 \mathrm{fs}$, full width at half maximum) is comparable to the pump (THz) pulse duration, the detected birefringence is a convolution between the $\mathrm{THz}$ intensity profile and optical intensity profile. In addition to the effect of the probe pulse duration, the fast change of the Gouy phase around our tightly focused beam leads to smearing out the fast oscillations of the $\mathrm{THz}$ pulse. Figure 1(c) shows the estimated $\Delta n$ in comparison with the simulation where $\Delta n$ is shown to follow the convolution between $I$ and a Gaussian probe pulse intensity profile, confirming the Kerr-like instantaneous nonlinearity in air. The maximum change of refractive index is $1.12 \times 10^{-7}$ corresponding to a reduction of the phase velocity of the optical pulse by $34 \mathrm{~m} / \mathrm{s}$ induced by the THz bullet. As the dispersion of our medium is negligible, the group velocity is reduced by the same amount.

Instantaneous Kerr phase retardation scales linearly with the excitation intensity. However, at extreme intensities, higher order nonlinear Kerr effects start playing a role, reversing the sign of the refractive index change. ${ }^{28,32}$ Such effects manifest themselves in the temporal and spectral
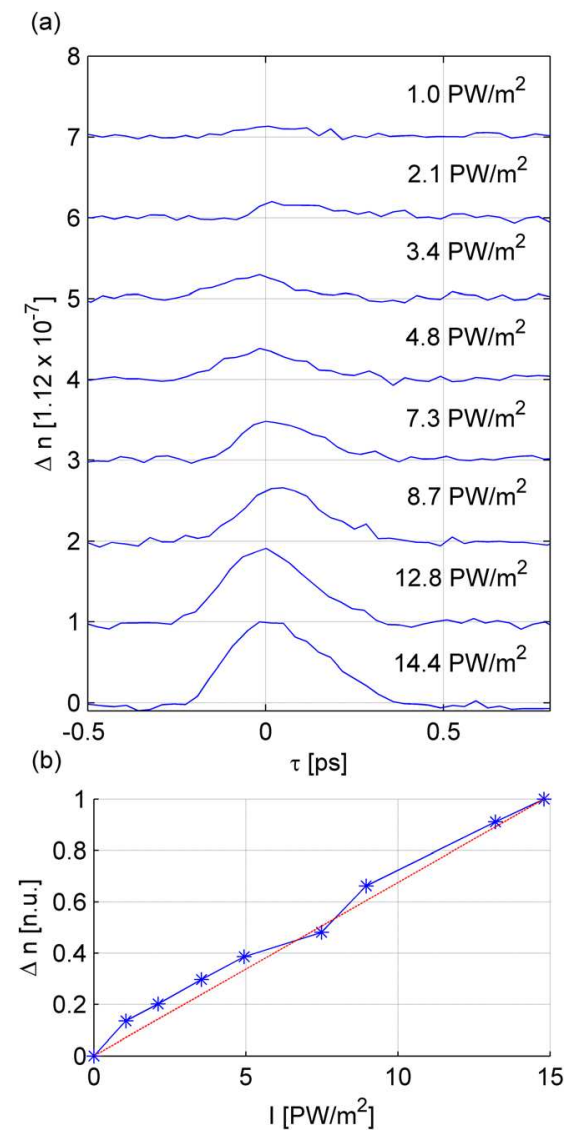

FIG. 2. (a) The dependence of the measured birefringence on the triggering THz peak intensity. (b) The birefringence is plotted against the THz peak fields (blue). A linear fit response (red) is also shown confirming $\Delta n=n_{2} I^{\mathrm{THz}}$ $=n_{2}\left|E^{\mathrm{THz}}\right| / 2 z_{0}$. 


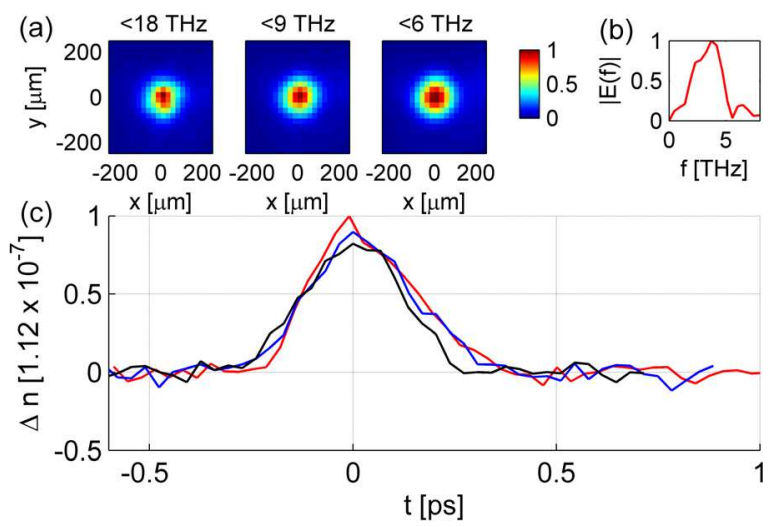

FIG. 3. (a) THz intensity spot at the focus for low pass filters with cut off frequencies at $18 \mathrm{THz}, 9 \mathrm{THz}$, and $6 \mathrm{THz}$, respectively. The corresponding average full width at half maxima are $85 \mu \mathrm{m}, 104 \mu \mathrm{m}$, and $117 \mu \mathrm{m}$. (b) The amplitude spectra of the exciting $\mathrm{THz}$ pulse before filtering. The peak spectral density occurs at $3.9 \mathrm{THz}$. (c) Frequency-dependent contribution of THz induced birefringence in air. Red, blue, and black lines correspond to the measurements done with low-pass filters cutting at $18 \mathrm{THz}, 9 \mathrm{THz}$, and 6 $\mathrm{THz}$, respectively.

responses as well as in violation of the linearity in $\Delta n=n_{2} I$. We verified that the excitation levels used here correspond to the linear Kerr regime by measuring the fluence dependence of the induced phase retardation. Figure 2(a) shows the extracted phase retardation for various excitation levels down to $6.9 \%$ of the maximum intensity. The time traces show no fluence-dependence change in the temporal response. The maximum phase retardation is shown in Fig. 2(b) versus the excitation field and manifests itself as a linear function of the THz peak intensity.

We next evaluate the Kerr nonlinear coefficient $n_{2}$. In comparison with the optical regime, a single cycle $\mathrm{THz}$ pulse carries a very broad spectrum of several octaves. As the considered electronic Kerr effect is instantaneous on the $\mathrm{THz}$ time scale and the dispersion of our nonlinear medium (dry air) is negligible, the nonlinear response (induced birefringence) is expected to be independent of the excitation frequency. In order to estimate which part of the spectrum contributes the most to the measured nonlinearity, we employ a set of low pass filters with cut off frequencies $\left(f_{\text {cutoff }}\right)$ at $\{18$; $9 ; 6 ; 3\} \mathrm{THz}$. The peak electric field and intensities was 3.3 $\mathrm{GV} / \mathrm{m}$ and $1.44 \mathrm{TW} / \mathrm{cm}^{2}$, respectively. The $\mathrm{THz}$ intensity profiles from these filters are shown in Fig. 3(a). The original (unfiltered) amplitude spectrum of the exciting pulse is shown in Fig. 3(b). Figure 3(c) shows the measured $\Delta n$ for different filters. In the case of $f_{\text {cutoff }}=3 \mathrm{THz}$, the field intensity leads to rotation below the sensitivity of our detection (Fig. 2(a)). Although the peak intensity obtained with the $<6 \mathrm{THz}$ LPF was $33 \%$ of the total intensity, the measured phase retardation was $74 \%$ of the maximum phase retardation which is attributed to the shorter Rayleigh length of high frequency components and the insensitivity of our relatively long probe pulse and spatially large probe size to high frequencies components. For simplicity, in the calculation of the Kerr coefficient, we considered only the spectral contents below $6 \mathrm{THz}$. From the measured phase retardation and assuming Gaussian beam characteristics around the focus, the Kerr nonlinear coefficient can thus be deduced as $1.3 \times 10^{-23} \mathrm{~m}^{2} / \mathrm{W}$ in the considered $\mathrm{THz}$ range. This value compares well with the measurements at the optical frequencies $\left(2.4 \times 10^{-23} \mathrm{~m}^{2} / \mathrm{W}\right)$.

In addition to the instantaneous effect, we observed evidence of retarded molecular alignment dynamics. Molecular orientation in gases induced by a THz pulse has been observed only in polar molecules (such as $\mathrm{N}_{2} \mathrm{O}$ (Ref. 25) and OCS ${ }^{23,24}$ ). Such molecules carry large permanent dipole moments (for example, $0.71 \mathrm{D}$ for OCS) which couple to an electromagnetic field via rotational transition frequencies. The orientation can thus be observed at relatively weak $\mathrm{THz}$ fields $(\approx 20 \mathrm{kV} / \mathrm{cm}$ for OCS). For non-polar molecules, however, much higher field strength $(\gg \mathrm{MV} / \mathrm{cm})$ is required for study of molecular alignment. Here, we demonstrate molecular alignment under such conditions using a strong $\mathrm{THz}$ transient.

Figure 4 shows the retarded $\mathrm{THz}$-induced adiabatic changes of the birefringence, observed on the time scale of tens of picoseconds, i.e., much longer than the stimulus pulse duration. The periodic change of birefringence originates from the off-resonant, THz-induced molecular alignment of the two principal air molecules $\mathrm{N}_{2}$ and $\mathrm{O}_{2}$ with quarter revival periods of $T_{N_{2}}^{1 / 4} \approx 2.1 \mathrm{ps}$ and $T_{O_{2}}^{1 / 4} \approx 2.9 \mathrm{ps}$, respectively.

(a)

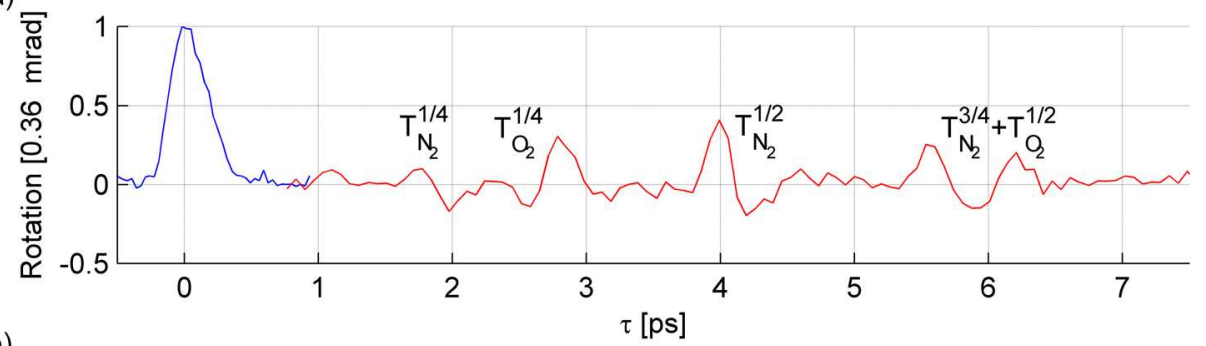

(b)

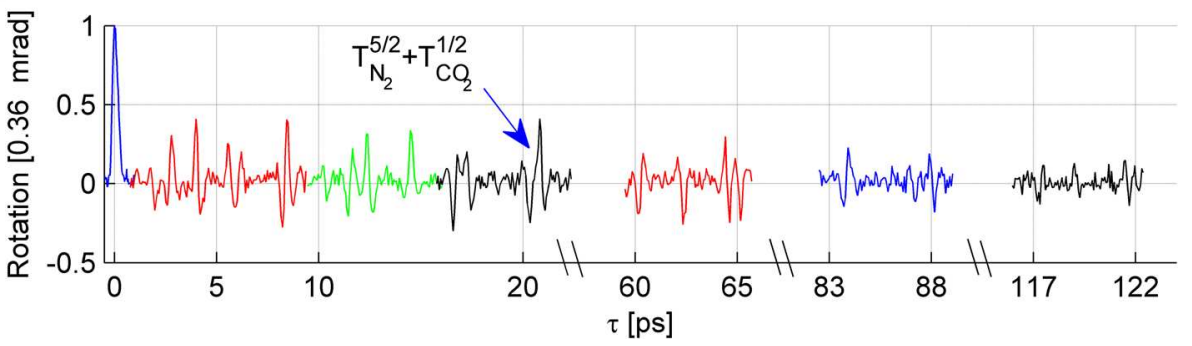

FIG. 4. (a) Induced birefringence initiated by the lambda-cubic $\mathrm{THz}$ pulse in air at ambient pressure and temperature. (b) Molecular alignment revivals for the non-polar $\mathrm{N}_{2}$ and $\mathrm{O}_{2}$ occur periodically at quarter-periods of 2.1 and $2.9 \mathrm{ps}$, respectively. At delay $\sim 21 \mathrm{ps}$, a trace of $1 / 2$ revival of $\mathrm{CO}_{2}$ is shown superimposed on $5 / 2$ revivals of the $\mathrm{N}_{2}$. At time zero, the THz electric field transient induces a non-permanent dipole moment which couples to the $\mathrm{THz}$ single-cycle electric field component. As the system coherence is maintained the revivals were recorded up to $120 \mathrm{ps}$. 
The short $\mathrm{THz}$ pulse induces a transient dipole moment in the molecules which couples to the $\mathrm{THz}$ electric field. In classical terms, the laser electric field exerts a torque on the molecules leading to alignment along the polarization axis of the laser. The coherent superposition of rotational levels dephases at a rate proportional to the square of the wavepacket width in J-space. Since coherence is maintained, the alignment revives at pre-determined times $\mathrm{T}_{\text {rev }}=1 / 2 \mathrm{Bc}$ with $\mathrm{B}$ the rotational constant and $\mathrm{c}$ the speed of light. At half of the period ( $4.15 \mathrm{ps}$ and $5.8 \mathrm{ps}$, respectively), the molecules are aligned perpendicular to the field. A maximum alignment is measured for the two first half-revivals $\left(\mathrm{N}_{2}\right)$. We observed revivals up to a delay of $120 \mathrm{ps}$, corresponding to the $15^{\text {th }}$ $\left(10^{\text {th }}\right)$ revival for $\mathrm{N}_{2}$ and $\mathrm{O}_{2}$. Moreover, around a delay $\sim 21$ ps, we trace $1 / 2$ revival period of $\mathrm{CO}_{2}$ (Ref. 33) superimposed on $5 / 2$ period of $\mathrm{N}_{2}$. The non-ionizing lambda-cubic $\mathrm{THz}$ alignment scheme presented here is capable of providing a substantial degree of alignment of non-polar molecules at room temperature and ambient pressure.

In conclusion, we presented quantitative measurement of low-frequency nonlinearities in air, triggered by an ultraintense lambda-cubic $\mathrm{THz}$ pulse. The experimental measurement of the instantaneous THz-induced Kerr nonlinearity coefficient $\mathrm{n}_{2}$ will support future modeling and experimentation of nonlinear propagation of $\mathrm{THz}$ in air such as $\mathrm{THz}$ Kerr self-focusing, THz self-phase modulation, and $\mathrm{THz}$ filamentation. On a delayed time scale, we observed the alignment of $\mathrm{N}_{2}, \mathrm{O}_{2}$, and $\mathrm{CO}_{2}$ and gave quantitative data on the THzinduced field-free alignment dynamics of non-polar air molecules. The revivals were initiated by a transient dipole moment induced by the strong THz pulse. Our approach represents a method for field-free alignment of non-polar molecules at intensities well below the ionization threshold which hold promise for enhanced control on molecular alignment.

We thank Gregor Knopp for fruitful discussions on molecular dynamics. We are grateful to Marta Divall, Alexandre Trisorio, and Andeas Dax for operating the Ti:sapphire laser system. The OPA operation was supported by Carlo Vicario and Marta Divall. The data acquisition software, motors control, and $\mathrm{THz}$ camera used in this work were previously installed by Carlo Vicario, Clemens Ruchert, Rasmus Ischebeck, Edwin Divall, and Balazs Monoszlai. We acknowledge financial support from the Swiss National Science Foundation (SNSF) (Grant No. 200021_146769). M.S. acknowledges partial funding from the European Community's Seventh Framework Programme (FP7/20072013) under Grant Agreement No. 290605 (PSI-FELLOW/ COFUND). C.P.H. acknowledges association to NCCRMUST and support from SNSF (Grant No. PP00P2_150732).
${ }^{1}$ C. Marceau, Y. Chen, F. Théberge, M. Châteauneuf, J. Dubois, and S. L. Chin, Opt. Lett. 34, 1417 (2009).

${ }^{2}$ C. P. Hauri, W. Kornelis, F. W. Helbing, A. Heinrich, A. Couairon, A. Mysyrowicz, J. Biegert, and U. Keller, Appl. Phys. B 79, 673 (2004).

${ }^{3}$ T. Kampfrath, K. Tanaka, and K. A. Nelson, Nat. Photonics 7, 680 (2013).

${ }^{4}$ C. Vicario, C. Ruchert, F. Ardana-Lamas, P. M. Derlet, B. Tudu, J. Luning, and C. P. Hauri, Nat. Photonics 7, 720-723 (2013).

${ }^{5}$ M. Shalaby, J. Fabianska, M. Peccianti, Y. Ozturk, F. Vidal, H. Sigg, R. Morandotti, and T. Feurer, Appl. Phys. Lett. 104, 171115 (2014).

${ }^{6}$ M. Liu, H. Y. Hwang, H. Tao, A. C. Strikwerda, K. Fan, G. R. Keiser, A. J. Sternbach, K. G. West, S. Kittiwatanakul, and J. Lu et al., Nature 487, 345 (2012).

${ }^{7}$ M. Mochizuki and N. Nagaosa, Phys. Rev. Lett. 105, 147202 (2010).

${ }^{8}$ M. Shalaby, F. Vidal, M. Peccianti, R. Morandotti, F. Enderli, T. Feurer, and B. Patterson, Phys. Rev. B 88, 140301(R) (2013).

${ }^{9}$ O. Schubert, M. Hohenleutner, F. Langer, B. Urbanek, C. Lange, U. Huttner, D. Golde, T. Meier, M. Kira, S. W. Koch, and R. Huber, Nat. Photonics 8, 119 (2014).

${ }^{10}$ M. Machholm and N. E. Henriksen, Phys. Rev. Lett. 87, 193001 (2001).

${ }^{11}$ M. Lemeshko, R. V. Krems, J. M. Doyle, and S. Kais, Mol. Phys. 111, 1648 (2013).

${ }^{12}$ R. Velotta, N. Hay, M. B. Mason, M. Castillejo, and J. P. Marangos, Phys. Rev. Lett. 87, 183901 (2001).

${ }^{13}$ J. Itatani, J. Levesque, D. Zeidler, H. Niikura, H. Pepin, J. C. Kieffer, P. B. Corkum, and D. M. Villeneuve, Nature 432, 867 (2004).

${ }^{14}$ H. Soifer, P. Botheron, D. Shafir, A. Diner, O. Raz, B. D. Bruner, Y. Mairesse, B. Pons, and N. Dudovich, Phys. Rev. Lett. 105, 143904 (2010).

${ }^{15}$ S. L. Chin, T.-J. Wang, C. Marceau, J. Wu, J. S. Liu, O. Kosareva, N. Panov, Y. P. Chen, J.-F. Daigle, S. Yuan et al., Laser Phys. 22, 1 (2012).

${ }^{16}$ J. Wu, Y. Tong, M. Li, H. Pan, and H. Zeng, Phys. Rev. A 82, 053416 (2010).

${ }^{17}$ H. J. Loesch and A. Remscheid, J. Chem. Phys. 93, 4779 (1990).

${ }^{18}$ J. Hajdu, Curr. Opin. Struct. Biol. 10, 569 (2000).

${ }^{19}$ R. Neutze, R. Wouts, D. van der Spoel, E. Weckert, and J. Hajdu, Nature 406, 752 (2000).

${ }^{20}$ A. G. York, Opt. Express 17, 13671 (2009).

${ }^{21}$ H. Stapelfeldt and T. Seideman, Rev. Mod. Phys. 75, 543 (2003).

${ }^{22}$ M. Shalaby and C. P. Hauri, Nat. Commun. 6, 5976 (2015).

${ }^{23}$ S. Fleischer, Y. Zhou, R. W. Field, and K. A. Nelson, Phys. Rev. Lett. 107, 163603 (2011).

${ }^{24}$ K. N. Egodapitiya, S. Li, and R. R. Jones, Phys. Rev. Lett. 112, 103002 (2014).

${ }^{25}$ H. Harde, S. Keiding, and D. Grischkowsky, Phys. Rev. Lett. 66, 1834 (1991).

${ }^{26}$ H. Y. Hwang, S. Fleischer, N. C. Brandt, B. G. Perkins, M. Liu, K. Fan, A. Sternbach, X. Zhang, R. D. Averitt, and K. A. Nelson, "A review of nonlinear terahertz spectroscopy with ultrashort tabletop-laser pulses," J. Mod. Opt. (published online).

${ }^{27} \mathrm{~N}$. Owschimikow, "Coherence in a thermal ensemble: Creation and decay of laser-induced alignment in nitrogen," Ph.D. thesis (Freie Universität Berlin, 2011).

${ }^{28}$ V. Loriot, E. Hertz, O. Faucher, and B. Lavorel, Opt. Express 17, 13429 (2009).

${ }^{29}$ A. Yariv, Optical Electronics in Modern Communications (Oxford University Press, Oxford, 1997).

${ }^{30}$ R. W. Boyd, Nonlinear Optics (Academic, San Diego, California, 1992).

${ }^{31}$ M. Zalkovskij, A. C. Strikwerda, K. Iwaszczuk, A. Popescu, D. Savastru, R. Malureanu, A. V. Lavrinenko, and P. U. Jepsen, Appl. Phys. Lett. 103, 221102 (2013).

${ }^{32}$ M. Bache, F. Eilenberger, and S. Minardi, Opt. Lett. 37, 4612 (2012).

${ }^{33}$ G. H. Lee, H. T. Kim, J. Y. Park, C. H. Nam, T. K. Kim, J. H. Lee, and H. Ihee, J. Korean Phys. Soc. 49, 337 (2006). 